\title{
Attenuated macrophage activation mediated by microRNA-183 knockdown through targeting NR4A2
}

\author{
FU-HAN GONG ${ }^{1}$, LI LONG $^{2}$, YONG-SHENG YANG ${ }^{1}$, DE-HONG SHEN ${ }^{1}$, YU-SONG ZHANG ${ }^{1}$, \\ XUE-SHENG WANG $^{1}$, XUE-PING ZHANG ${ }^{1}$ and XIAO-QIANG XIAO ${ }^{1}$ \\ Departments of ${ }^{1}$ Cardiology and ${ }^{2}$ Clinical Laboratory, Tongren Municipal People's Hospital, \\ Tongren, Guizhou 554300, P.R. China
}

Received July 6, 2020; Accepted December 30, 2020

DOI: $10.3892 / \mathrm{etm} .2021 .9731$

\begin{abstract}
Atherosclerosis is considered a chronic inflammatory disease, and macrophages function as important mediators in the development of atherogenesis. MicroRNA (miR)-183 is a small non-coding RNA that acts as a novel tumor suppressor and has recently been proposed to affect cardiac hypertrophy. However, the exact role and underlying mechanism of miR-183 in macrophage activation remain unknown. In the present study, miR-183 showed upregulated expression in atheromatous plaques and in bone marrow-derived macrophages (BMDMs) subjected to stimulation with oxidized low-density lipoproteins. Using a miR-183 loss-of-function strategy, it was demonstrated that miR-183 knockdown significantly increased resolving M2 macrophage marker expression but decreased proinflammatory M1 macrophage marker expression, as well as attenuated $\mathrm{NF}-\kappa \mathrm{B}$ activation. Moreover, decreased foam-cell formation accompanied by upregulation of genes involved in cholesterol efflux and downregulation of genes implicated in cholesterol influx was found in BMDMs transfected with a miR-183 inhibitor. Mechanistically, macrophage activation mediated by miR-183 silencing was partially attributed to direct upregulation of NR4A2 expression in BMDMs. Thus, the present study suggests that neutralizing miR-183 may be a potential therapeutic strategy for the treatment of atherosclerosis.
\end{abstract}

\section{Introduction}

Atherosclerosis is well recognized as a chronic inflammatory disease (1) that can cause various cardiovascular pathologies, including myocardial infarction, sudden cardiac death and stroke (2). During the development of atherosclerosis,

Correspondence to: Dr Xiao-Qiang Xiao, Department of Cardiology, Tongren Municipal People's Hospital, 120 Taoyuan Avenue, Chengguan, Tongren, Guizhou 554300, P.R. China E-mail:2837124871@qq.com

Key words: atherosclerosis, microRNA-183, macrophage, inflammation, NR4A2 infiltrated macrophages triggered by endothelial dysfunction play an important role (3). Macrophage polarization refers to an estimate of macrophage activation at a given point in space and time. Currently, the macrophage field has arrived at a partial consensus to describe the broad grouping of macrophage activation phenotypes and the underlying mechanisms (4). The classical inflammatory macrophage phenotype has been termed the M1 phenotype and is usually induced by a combination of interferon- $\gamma$ and the Toll-like receptor 4 ligand lipopolysaccharide. By contrast, the alternatively activated macrophage populations are termed M2 populations and can be induced by incubation of macrophages with interleukin (IL)-4 and IL-13. Upon exposure to multiple environmental stimuli, macrophages exhibit considerable plasticity, which is responsible for the inflammatory response underlying atherogenesis through a switch between classically activated M1 macrophages, which produce a wide variety of proinflammatory mediators, and alternatively activated M2 macrophages that alleviate inflammation (5-7). Additionally, lesion macrophages can give rise to lipid-laden foam-cell formation following excess engulfment of oxidized low-density lipoprotein (Ox-LDL) $(8,9)$. Importantly, previous studies have also suggested that M1 or M2 macrophages may be the main foam cell precursors through the regulation of cholesterol transport by a liver-X-receptor-dependent mechanism $(10,11)$. Macrophage activation constituted by the aforementioned macrophage polarization shift and foam-cell formation leads to the enlargement of necrotic cores and formation of vulnerable plaques (12). Thus, exploring the key regulators and potential mechanisms of atherosclerosis-associated macrophage activation may provide clinicians with an effective strategy for preventing atherosclerosis.

MicroRNAs (miRNAs/miRs) are an evolutionarily conserved class of small non-coding RNAs with 20-23 nucleotides (13) and exert important regulatory effects on various biological processes, including cell development, cell differentiation, cell proliferation, apoptosis and immune inflammation, through regulation of gene expression by directly binding to the 3'-untranslated regions (3'-UTRs) of their target mRNAs (14-16). Accumulating evidence has demonstrated that miR-183 is strongly implicated and functions in various aspects of tumorigenesis. miR-183 is reported to be a potent prognostic marker for lung cancer through 
negative regulation of the metastatic potential of lung cancer cells but acts as a tumor suppressor in human non-small cell lung cancer by downregulating MTA1 (17). miR-183 inhibits osteosarcoma cell growth and invasion by regulating the LRP6-Wnt/ $\beta$-catenin signaling pathway (18), and promotes proliferation and invasion in esophageal squamous cell carcinoma by targeting programmed cell death 4 (19). Recently, miR-183 has emerged as an important player in cardiovascular disease and showed downregulated expression in hypertrophic cardiac tissues subjected to transverse aortic constriction surgery (20). Additionally, inhibition of miR-183 expression in HUVECs with Ox-LDL-induced injury can enhance cell activity, decrease inflammation level, and thus prevent cell injury by increasing insulin receptor substrate 1 expression (21). miR-183 alleviates early injury, including decreasing brain edema, neurobehavioral defects, inflammation, oxidative stress and iron deposition, and by inhibiting heme oxygenase-1 expression after intracerebral hemorrhage. A miR-183 inhibitor was also found to suppress anaphylaxis and atopic dermatitis by decreasing NF- $\mathrm{BB}$ activation (22). However, the potential role and precise underlying mechanism of miR-183 in macrophage activation have not been elucidated.

The present study demonstrated that significantly upregulated miR-183 expression was found in atherosclerotic plaques of $\mathrm{ApoE}^{-/-}$mice fed a high-fat diet (HFD) and in BMDMs upon Ox-LDL stimulation. miR-183 knockdown dramatically accelerated the activation of resolving M2-polarized macrophages but attenuated the activation of classical M1-polarized macrophages, as well as inhibited NF- $\mathrm{NB}$ activation. Additionally, miR-183 silencing attenuated foam-cell formation accompanied by increased upregulation of genes involved in cholesterol efflux, and downregulation of genes implicated in cholesterol influx. Mechanistically, it was verified that NR4A2 was the direct target for the attenuating effect on macrophage activation regulated by miR-183 knockdown. Overall, the present study results demonstrated that blockade of the miR-183-NR4A2 axis might be a promising strategy for the regulation of macrophage activation implicated in atherogenesis.

\section{Materials and methods}

Animals and morphological analysis. The animal study procedures were performed in accordance with the National Institutes of Health Guide for the Care and Use of Laboratory Animals and were approved by the Animal Care and Use Committee of the Tongren municipal people's Hospital. A total of 25 male ApoE deficient mice (ApoE ${ }^{-/-}$; age, 8-12 weeks; weight, 21-28 g; Beijing Zhongke Biotechnology Co., Ltd.) were fed a high-fat diet (HFD; $15.8 \%$ fat and $1.25 \%$ cholesterol) or normal chow (NC) purchased from Beijing Huafukang Bioscience Co Inc., respectively, for up to 12 weeks. Animals were maintained under a $12 \mathrm{~h}$ light/dark cycle with free access to food and water at a controlled temperature of $22-26^{\circ} \mathrm{C}$ and relative humidity $(65 \pm 10 \%)$. Hearts from mice subjected to NC or HFD treatment for 12 weeks were excised after anesthetization via the intraperitoneal injection of pentobarbital sodium $(50 \mathrm{mg} / \mathrm{kg})$. The tissues were subsequently dehydrated at room temperature in $85 \%$ alcohol for $15 \mathrm{~min}, 95 \%$ alcohol twice for $10 \mathrm{~min}$ each and in $100 \%$ alcohol twice for $15 \mathrm{~min}$ and embedded in paraffin for histological analysis. Consecutive
5- $\mu \mathrm{m}$ sections of the atrioventricular valve region of each heart were collected and stained with hematoxylin and eosin (H\&E) for $5 \mathrm{~min}$ for morphological analysis at a temperature of $26^{\circ} \mathrm{C}$. Samples were observed under a light microscope at a x40 magnification.

Cell culture and small interfering (si)RNA transfection. Approximately $5 \times 10^{7}$ nucleated BMDMs isolated form femurs and tibias of ApoE-mice were cultured in $10 \mathrm{ml}$ of RPMI with $10 \%$ fetal bovine serum and Macrophage Colony-Stimulating Factor (MCSF; 50 ng/ml; cat. no. M9170, Sigma-Aldrich; Merck KGaA) (23-25). Cells were cultured in RPMI-1640 (Sigma-Aldrich; Merck KGaA) containing $10 \%$ fetal bovine serum (Sigma-Aldrich; Merck KGaA) and $1 \%$ penicillin-streptomycin. The synthetic miR-183 antagomir was synthesized by GenePharma. BMDMs were transfected with miR-183 antagomir and the corresponding controls (NC) at a concentration of $50 \mathrm{nM}$ using a riboFEC CP Transfection kit according to the manufacturer's protocol. The infected BMDMs stimulated with $15 \mathrm{ng} / \mathrm{ml}$ Ox-LDL, for $24 \mathrm{~h}$ after serum starvation for one day, were utilized for the in vitro functional study.

Reverse transcription-quantitative (RT-q)PCR and western blotting. Total mRNA was extracted by trichloromethane, dissolved in DEPC-water, and then reverse transcribed into cDNA with a Transcriptor First Strand cDNA Synthesis kit (F. Hoffmann-La Roche AG) as previously described (26). The expression levels of the target genes were quantified by real-time PCR using LightCycler 480 SYBR Green 1 Master mix and a LightCycler 480 QPCR System (F. Hoffmann-La Roche AG). The thermocycling conditions were as follows: $95^{\circ} \mathrm{C}$ for $10 \mathrm{~min}$ followed by 50 cycles of $95^{\circ} \mathrm{C}$ for $10 \mathrm{sec}, 55^{\circ} \mathrm{C}$ for $10 \mathrm{sec}, 72^{\circ} \mathrm{C}$ for $5 \mathrm{sec} ; 99^{\circ} \mathrm{C}$ for $1 \mathrm{sec} ; 59^{\circ} \mathrm{C}$ for $15 \mathrm{sec} ; 95^{\circ} \mathrm{C}$ for $1 \mathrm{sec}$; and cooling at $40^{\circ} \mathrm{C}$. The relative transcription levels of the target genes were normalized against GAPDH, while the level of miR-375 was normalized to U6 level. The $2^{-\Delta \Delta \mathrm{Cq}}$ method was used (27). The primers are listed in the Table I.

Protein concentrations were determined by Bicinchoninic Acid Kit assay (cat. no. BCA1; Sigma-Aldrich; Merck KGaA). The proteins $(5 \mu \mathrm{g})$ were separated by $7.5 \%$ SDS-PAGE (Invitrogen; Thermo Fisher Scientific, Inc.) and then transferred to polyvinylidene fluoride membranes, which were blocked in Tris-buffered saline (TBS) and Tween-20 containing $5 \%$ non-fat milk $1 \mathrm{~h}$ at room temperate. Subsequently, the membranes were incubated with the appropriate primary antibodies overnight at $4^{\circ} \mathrm{C}$.

The membranes were then incubated with a secondary IRDye ${ }^{\circledR} 800 \mathrm{CW}$-conjugated antibody $(1: 5,000$; cat. no. ab216773; Abcam) for $1 \mathrm{~h}$ at $37^{\circ} \mathrm{C}$, and treated with enhanced chemiluminescence reagent (Thermo Fisher Scientific, Inc.) before being visualized with Molecular Imager ChemiDoc ${ }^{\mathrm{TM}}$ XRS (Bio-Rad Laboratories, Inc.) using Image Lab ${ }^{\mathrm{TM}}$ Software 5.1 (Bio-Legend Scientific Co., Ltd). The expression levels of specific proteins were normalized against GAPDH expression. The antibodies used are listed in the Table II.

Foam-cell formation. BMDMs serum-starved for $24 \mathrm{~h}$ and stimulated with $15 \mathrm{ng} / \mathrm{ml} \mathrm{Ox-LDL}$ for $24 \mathrm{~h}$ were collected. To 
Table I. Sequences of primers used for reverse transcription-quantitative PCR.

\begin{tabular}{|c|c|}
\hline Primer & Sequence, $5^{\prime}$ to $3^{\prime}$ \\
\hline$T N F-\alpha-F$ & ATGGCCTCCCTCTCATCAGT \\
\hline$T N F-\alpha-\mathrm{R}$ & ATAGCAAATCGGCTGACGGT \\
\hline $\operatorname{Inos}-\mathrm{F}$ & AGGGCCACCTCTACATTTGC \\
\hline $\operatorname{Inos}-\mathrm{R}$ & TGCCCCATAGGAAAAGACTGC \\
\hline$C O X-2-\mathrm{F}$ & ATTGCCCTCCССТСТСТАCG \\
\hline$C O X-2-\mathrm{R}$ & CGGCTCATGAGTGGAGAACG \\
\hline$I L-6-\mathrm{F}$ & CTGGAGTACCATAGCTACCTGG \\
\hline$I L-6-\mathrm{R}$ & ACTCCTTCTGTGACTCCAGC \\
\hline Arg-1-F & AAAGGCCGATTCACCTGAGC \\
\hline Arg-1-R & AGGTAGTCAGTCCCTGGCTT \\
\hline$T G F-\beta-\mathrm{F}$ & AGAGCCCTGGATACCAACTATTG \\
\hline$T G F-\beta-\mathrm{R}$ & TGCGACCCACGTAGTAGACG \\
\hline$M r c-1-\mathrm{F}$ & CCTGTAACTACACACTCATCCA \\
\hline$M r c-1-\mathrm{R}$ & CATTCTGCTCGATGTTGCCC \\
\hline$P P A R \gamma-\mathrm{F}$ & GCTTGTGAAGGATGCAAGGG \\
\hline$P P A R \gamma-\mathrm{R}$ & GATATCACTGGAGATCTCCGCC \\
\hline$S R-A-\mathrm{F}$ & TGGAGGAGAGAATCGAAAGCA \\
\hline$S R-A-\mathrm{R}$ & CTGGACTGACGAAATCAAGGAA \\
\hline$C D 36-\mathrm{F}$ & GACTGGGACCATTGGTGATGA \\
\hline$C D 36-\mathrm{R}$ & AAGGCCATCTCTACCATGCC \\
\hline$A B C A 1-\mathrm{F}$ & AGGCACTCAAGCCACTGCTTGT \\
\hline$A B C A 1-\mathrm{R}$ & TGCCTCTGCTGTCTAACAGCGT \\
\hline$A B C G 1-\mathrm{F}$ & GGTTGCGACATTTGTGGGTC \\
\hline$A B C G 1-\mathrm{R}$ & TTCTCGGTCCAAGCCGTAGA \\
\hline$N R 4 A 2-\mathrm{F}$ & GCCATGCCTTGTGTTCAGGCGCAG \\
\hline$N R 4 A 2-\mathrm{R}$ & GGAGGTCTTAGAAAGGTAAAGTGTCC \\
\hline$G A P D H-\mathrm{F}$ & GGTGGACCTCATGGCCTACA \\
\hline$G A P D H-\mathrm{R}$ & СТCTCTTGCTCTCAGTATCCTTGCT \\
\hline$U 6-\mathrm{F}$ & CTCGCTTCGGCAGCACA \\
\hline U6-R & AACGCTTCACGAATTTGCGT \\
\hline
\end{tabular}

F, forward; $R$, reverse.

visualize lipid droplets by oil red $\mathrm{O}$ staining, the cells were washed twice with PBS and fixed with $4 \%$ paraformaldehyde for $10 \mathrm{~min}$ at temperature of $26^{\circ} \mathrm{C}$. The slides were rinsed quickly in $60 \%$ isopropyl alcohol and stained in $0.3 \%$ oil red $\mathrm{O}$ solution for $10 \mathrm{~min}$, respectively. Subsequently, the slides were washed briefly in 60\% isopropyl alcohol for 3-10 sec and mounted using $50 \%$ glycerol, which were observed under a light microscope (magnification, x200).

Luciferase activity. Luciferase reporters were generated based on Pezx-MT01 vector purchased from Shanghai GenePharma Co., Ltd. HEK293 cells were purchased from the Type Culture Collection of the Chinese Academy of Sciences and cultured at $37^{\circ} \mathrm{C}$ in a humidified $5 \% \mathrm{CO}_{2}$ incubator. Cells were seeded into a 24 -well plate and infected with miR-183 antagomir $(50 \mathrm{~nm})$ or $\mathrm{NC}(50 \mathrm{~nm})$ and then transfected with luciferase reporter containing the wild-type or mutant 3'-UTR of NR4A2 using Lipofectamine ${ }^{\circledR} 2000$
(Invitrogen; Thermo Fisher Scientific, Inc.) for $48 \mathrm{~h}$ according to the manufacturer's instructions. Then, dual luciferase assays (Promega Corporation) was performed to analysis the luciferase activity according to the manufacturer's instructions.

TargetScan. TargetScan predicts the biological targets of miRNAs by searching for the presence of conserved $8 \mathrm{mer}$, $7 \mathrm{mer}$ and $6 \mathrm{mer}$ sites that match the seed region of each miRNA. The URL of the database was http://www.targetscan. org/mmu_71/.

Statistical analysis. All statistical data were analyzed using SPSS software version 22.0 (IBM Corp.) and are presented as the mean \pm SD. Differences between two groups were analyzed by independent-samples t-tests, while differences among multiple groups were analyzed by one-way ANOVA followed by a Bonferroni post hoc test or with Tamhane's T2 analysis. $\mathrm{P}<0.05$ was considered to indicate a statistically significant difference.

\section{Results}

miR-183 expression is upregulated in atheromatous plaques and macrophages. To investigate the involvement of miR-183 in atherogenesis, atherosclerotic plaques from $\mathrm{ApoE}^{-/-}$mice induced by HFD feeding were first examined to determine whether miR-183 expression was altered. Hematoxylin and eosin staining demonstrated the typical pathological changes of atherosclerotic plaques in $\mathrm{ApoE}^{-/}$mice fed a HFD for 12 weeks, characterized by the thin fibrous cap and large necrotic core (Fig. 1A). RT-Qpcr analysis revealed that miR-183 Mrna expression levels were markedly upregulated in arteries of HFD-fed $\mathrm{ApoE}^{-/}$mice compared with those of NC-treated mice (Fig. 1A). Furthermore, the expression of miR-183 in BMDMs subjected to Ox-LDL stimulation was also evaluated. It was found that miR-183 expression was gradually upregulated in BMDMs following Ox-LDL stimulation (Fig. 1B). These findings suggest that miR-183 is involved in the development of atherosclerosis, partially through functioning in macrophages.

miR-183 silencing ameliorates the inflammatory response. Given the significant change in miR-183 expression in atheromatous plaques and macrophages treated with Ox-LDL, miR-183 loss-of-function experiments were performed in macrophages. It was observed that miR-183 expression was notably decreased in BMDMs following miR-183 inhibitor transfection (Fig. 2A). Furthermore, the Mrna levels of genes associated with proinflammatory M1-polarized macrophages, including tumor necrosis factor- $\alpha$, inducible nitric oxide synthase, cyclooxygenase 2 and IL-6, were dramatically downregulated in BMDMs transfected with the miR-183 inhibitor upon Ox-LDL treatment compared with the control group (Fig. 2B). In contrast, the Mrna expression levels of genes associated with resolving M2-polarized macrophages, including Arginase-1 (Arg-1), transforming growth factor- $\beta$, mediator of replication checkpoint protein 1 and peroxisome proliferator-activated receptors $\gamma$ were significantly upregulated in miR-183 inhibitor-transfected BMDMs with Ox-LDL treatment compared with control cells (Fig. 2C). Moreover, 
Table II. Antibodies for immunoblot analyses.

\begin{tabular}{lllll}
\hline Antibody & Catalogue number & Dilution & \multicolumn{1}{c}{ Supplier } & Sources of species \\
\hline NR4A2 & 10975-2-AP & $1: 200$ & ProteinTech Group, Inc. & Rabbit \\
IL-6 & AF-406-NA & $1: 200$ & R\&D Systems, Inc. & Goat \\
Arg-1 & 610708 & $1: 200$ & BD Biosciences & Mouse \\
CD36 & sc-7309 & $1: 100$ & Santa Cruz Biotechnology, Inc. & Mouse \\
ABCA1 & ab7360 & $1: 200$ & Abcam & Rabbit \\
P-p65 & ab76302 & $1: 200$ & Abcam & Rabbit \\
P65 & ab16502 & $1: 100$ & Abcam & Rabbit \\
GAPDH & $21181: 1,000$ & Cell Signaling Technology, Inc. & Rabbit \\
\hline
\end{tabular}

A

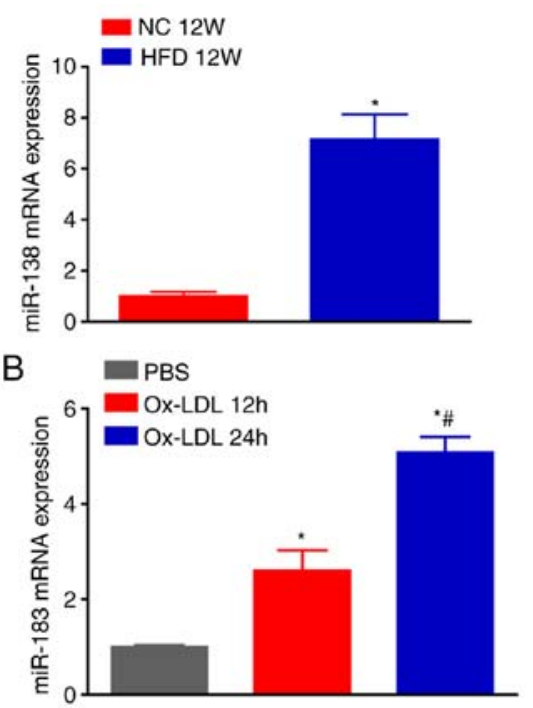

Figure 1. miR-183 expression in atherosclerotic plaques and macrophages. (A) Left: Aortic roots from ApoE ${ }^{-/-}$mice treated with normal chow or a HFD for 12 weeks were stained with hematoxylin and eosin. Scale bar, $500 \mu \mathrm{m}$. Right: Qpcr analysis of miR-183 Mrna expression in the entire aorta in ApoE ${ }^{-/-}$mice treated with $\mathrm{NC}$ for 12 weeks or with HFD for 12 weeks. $\mathrm{n}=3$. ${ }^{*} \mathrm{P}<0.05$ vs. ApoE ${ }^{-/}$with NC-12W treatment group. (B) RT-PCR analysis of miR-183 Mrna expression in bone marrow-derived macrophages stimulated with PBS and Ox-LDL for 12 or $24 \mathrm{~h} .{ }^{*} \mathrm{P}<0.05$ vs. PBS-treated group. ${ }^{\#} \mathrm{P}<0.05$ vs. Ox-LDL treated for $12 \mathrm{~h}$ group. miR, microRNA; RT-PCR, reverse transcription-quantitative PCR; HFD, high-fat diet; Ox-LDL, oxidized low-density lipoprotein; NC, negative control.

IL-6 and Arg-1 protein levels determined by western blotting analysis were consistent with the Mrna expression levels (Fig. 2D). Furthermore, phosphorylation of p65, an important event in the activation of the inflammatory mediator nuclear factor-kappa B (NF- $\kappa \mathrm{B})$, was decreased in BMDMs transfected with the miR-183 inhibitor (Fig. 2E).

miR-183 knockdown attenuates foam-cell formation. After excess engulfment of Ox-LDL, macrophages form foam cells, which play an important role in atherogenesis. A significant decrease in foam-cell formation in BMDMs transfected with the miR-183 inhibitor was observed upon Ox-LDL stimulation, as evaluated by neutral lipid staining with oil red $\mathrm{O}$, compared with the control group (Fig. 3A). Subsequently, the markers associated with cholesterol uptake and efflux that contribute to foam-cell formation were evaluated. BMDMs transfected with miR-183 inhibitor presented with decreased Mrna levels of genes (SR-A and CD36) involved in cholesterol influx, whereas the expression levels of markers implicated in cholesterol efflux (ABCA1 and ABCG1) were increased (Fig. 3B).
Moreover, the CD36 and ABCA1 protein levels determined by western blot analysis were consistent with the Mrna expression levels (Fig. 3C).

Knockdown of miR-183 promotes NR4A2 expression. Next, bioinformatic analysis using the program TargetScan was performed to elucidate the mechanism by which miR-183 affects macrophage activation. The results indicated that NR4A2 contained a putative binding site for miR-183 in its 3'UTR (Fig. 4A). Moreover, luciferase reporter assays demonstrated that miR-183 inhibitor transfection promoted NR4A2 luciferase activity, whereas the above effect was abolished when the predicted binding sites within the NR4A2 3'UTR were mutated (Fig. 4B). As expected, NR4A2 showed significantly upregulated Mrna and protein expression in BMDMs transfected with the miR-183 inhibitor (Fig. 4C and D). Collectively, these findings suggested that the effects of miR-183 knockdown on macrophage activation were partially mediated by the upregulated expression of its molecular target, NR4A2. 
A

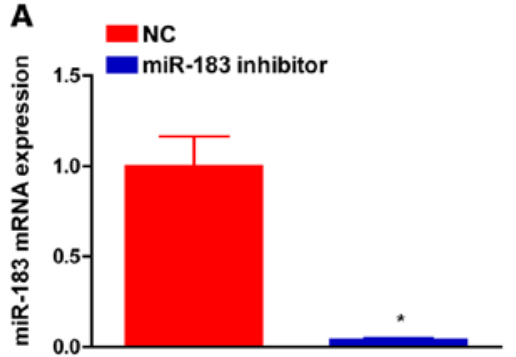

D
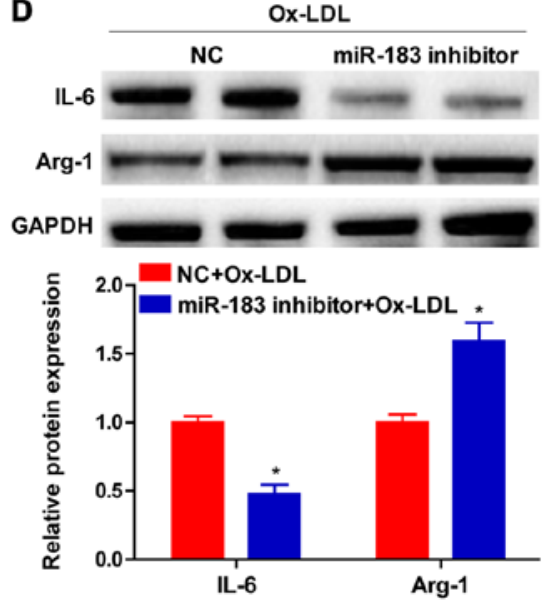

B

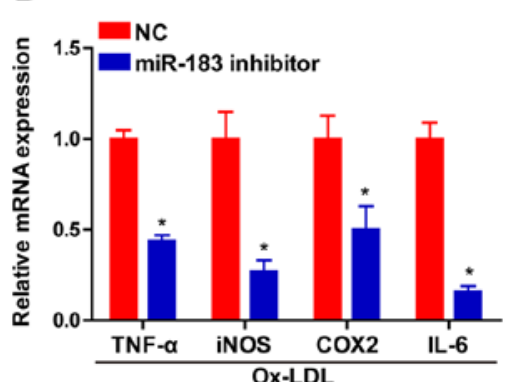

C

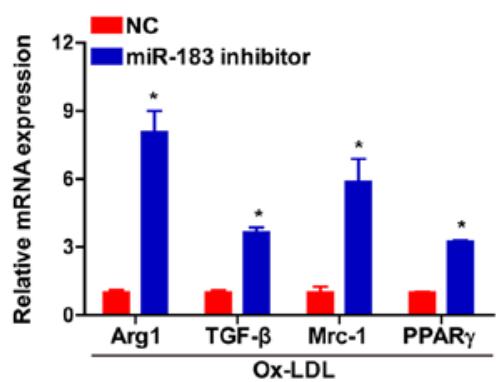

E

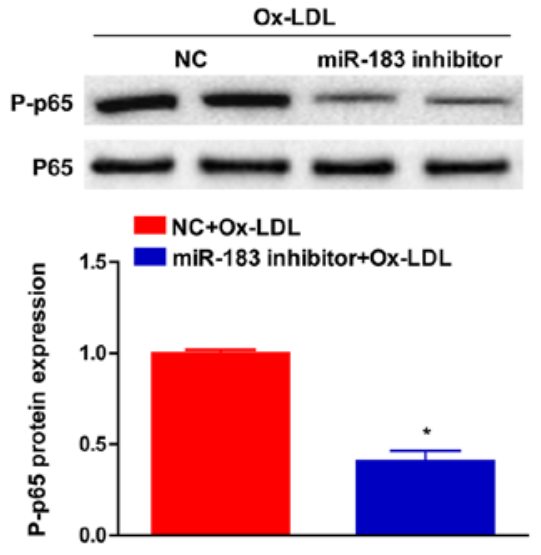

Figure 2. Pro-inflammatory macrophage response regulated by miR-183 knockdown. (A) miR-183 expression in BMDMs infected with miR-183 inhibitor or NC. Mrna expression of M1 marker genes (B) and M2 marker genes (C) in BMDMs transfected with miR-183 inhibitor or NC examined by reverse transcription-quantitative PCR analysis. (D and E) The protein level of IL-6 and Arg-1, as well as phosphorylated and total p65 in BMDMs infected with miR-183 inhibitor or $\mathrm{NC}$ tested by western blot analysis. "P<0.05 vs. control group. miR, microRNA; BMDMs, bone marrow-derived macrophages; $\mathrm{NC}$, negative control; $\mathrm{TNF} \alpha$, tumor necrosis factor $\alpha$; Inos, inducible nitric oxide synthase; COX, cyclooxygenase; IL, interleukin; Ox-LDL, oxidized low-density lipoprotein; Arg, arginase; TGF, transforming growth factor; Mrc, mediator of replication checkpoint protein 1; PPAR, peroxisome proliferator-activated receptors.

A

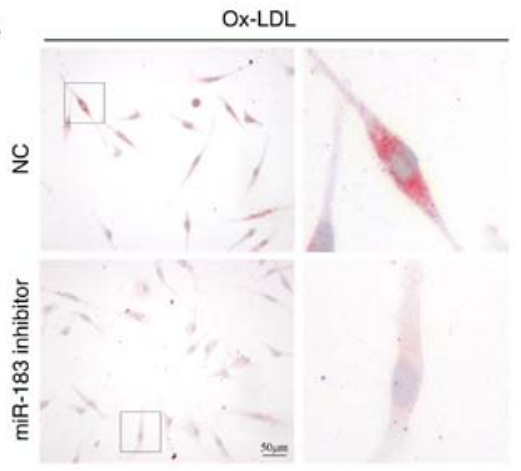

C

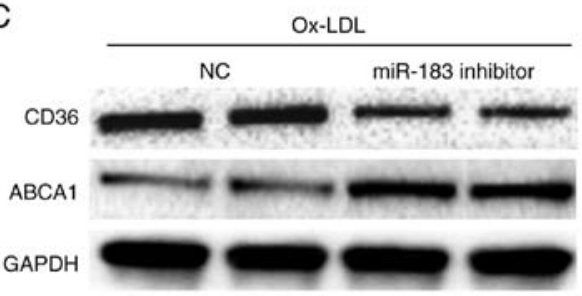

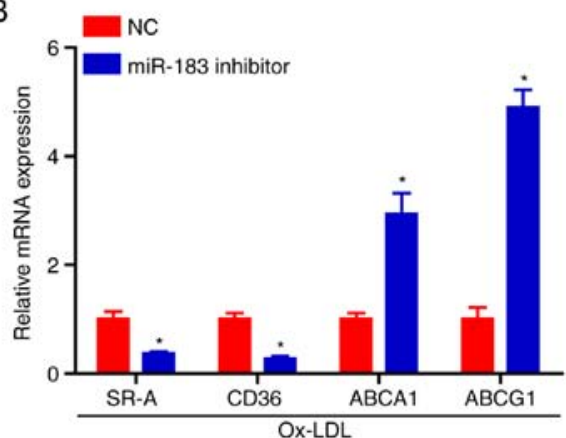

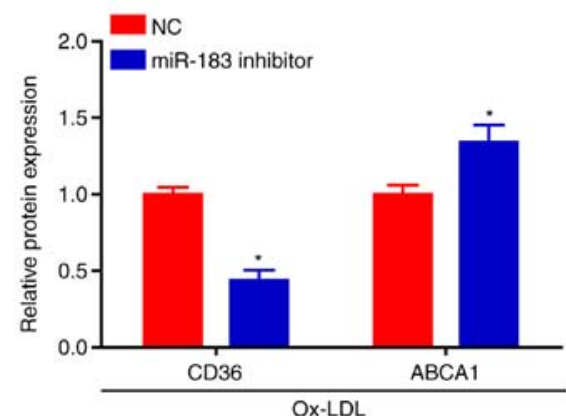

Figure 3. miR-183 silencing ameliorates foam-cell formation. (A) Foam-cell formation in macrophages infected with miR-183 inhibitor or NC induced by Ox-LDL. Scale bar, $50 \mu \mathrm{m}$. (B) The Mrna expression of markers associated with cholesterol uptake ability (CD36 and SR-A) and cholesterol efflux (ABCA1 and ABCG1) in BMDMs infected with miR-183 inhibitor or NC induced by Ox-LDL. (C) The protein expression levels of CD36 and ABCA1 in BMDMs infected with miR-183 inhibitor or NC. ${ }^{*} \mathrm{P}<0.05$ vs. control group. miR, microRNA; BMDMs, bone marrow-derived macrophages; Ox-LDL, oxidized low-density lipoprotein; $\mathrm{NC}$, negative control. 
A

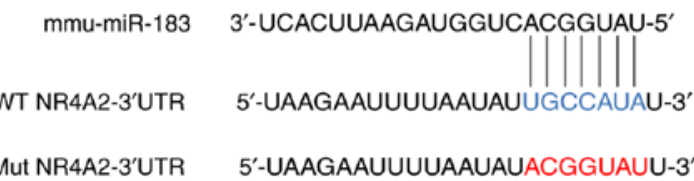

B

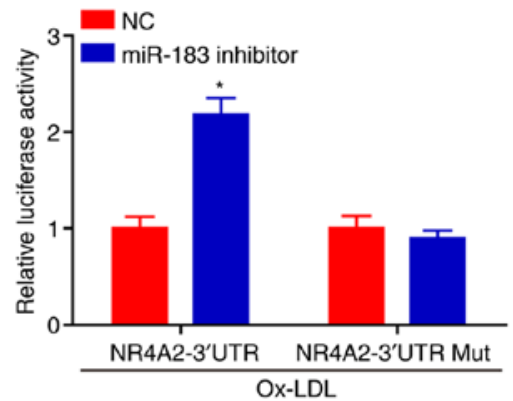

C

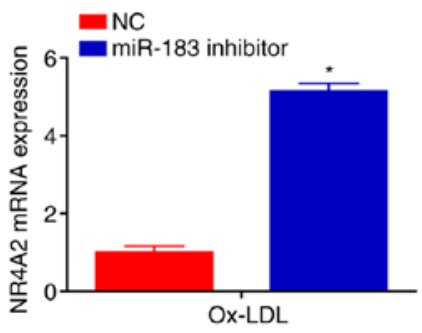

D

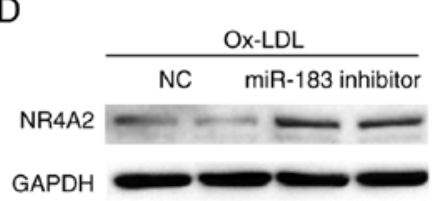

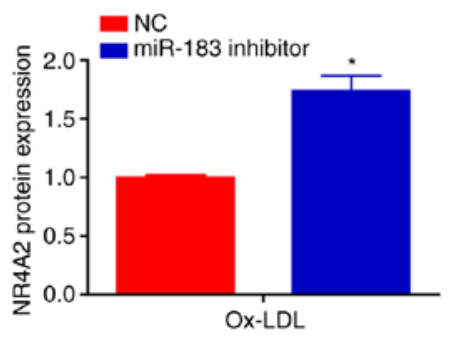

Figure 4. miR-183 targets to NR4A2. (A) Potential target sites for miR-183 in the 3'UTR of murine NR4A2 Mrna. (B) Luciferase reporter assays in HEK293 cells treated with a miR-183 inhibitor or NC using a Pezx-MT01 vector containing the NR4A2-3'UTR or the NR4A2-3'UTR with mutations in the predicted miR-183 binding site. (C and D) Quantitative PCR and western blot analysis of NR4A2 expression in bone marrow-derived macrophages infected with miR-183 inhibitor or NC stimulated with Ox-LDL. "P<0.05 vs. NC group. miR, microRNA; UTR, untranslated region; WT, wild type; Mut, mutant; NC, negative control; Ox-LDL, oxidized low-density lipoprotein.

\section{Discussion}

The present study first demonstrated that miR-183 is a novel and independent indicator of macrophage activation implicated in atherogenesis, which exhibited an upregulated expression in atherosclerotic plaques of $\mathrm{ApoE}^{-/-}$mice fed with HFD and BMDMs with Ox-LDL stimulation. The loss-of-function strategy demonstrated that miR-183 knockdown promoted M2 phenotypic macrophage but attenuated M1 macrophage switching. Meanwhile, miR-183 knockdown significantly ameliorated foam cell forming by increased cholesterol efflux but decreased cholesterol uptake. Mechanistically, NR4A2 was the direct target of miR-183 and the silencing of miR-183 dramatically promoted NR4A2 expression. On the basis of the results from the present study, an unexplored role of miR-183 in regulation of macrophage activation was identified, which is at least partially through regulation of NR4A2 expression.

Over the past decade, multiple studies have demonstrated that various miRNAs are widely involved in numerous human disorders and are especially implicated in multiple cardiometabolic diseases, such as obesity, diabetes, heart failure and atherosclerosis; the collective evidence implies their potential as therapeutic targets and provides a new direction for the study of these diseases $(15,28-34)$. Atherosclerosis is recognized as a complex multifactorial pathological process (35), and microRNAs have emerged as important regulators of atherosclerosis development through the regulation of the inflammatory response via the switch between classical proinflammatory M1 macrophage activation and alternative inflammation-resolving M2 macrophage activation (36-39). In the present study, it was observed that the expression of miR-183 was gradually upregulated in atherosclerotic plaques of $\mathrm{ApoE}^{-1-}$ mice and in BMDMs following Ox-LDL stimulation. miR-183 silencing attenuated the Mrna expression of prototypical genes associated with proinflammatory M1-polarized macrophages but increased the Mrna expression levels of genes associated with anti-inflammatory M2-polarized macrophages. The predominance of M2-polarized macrophages in bladder cancer affects angiogenesis, the tumor grade and invasiveness. Macrophage polarization has been well recognized to exert an important role in the regulation of tumorigenesis and angiogenesis (40), and has emerged as the main precursor of foam-cell formation mediated by macrophages, which together play important roles in the development of atherosclerosis. The present study demonstrated that a miR-183 inhibitor attenuated the formation of foam cell contaminants, with decreased Mrna levels of genes involved in cholesterol influx but increased expression levels of markers implicated in cholesterol efflux.

miRNAs are capable of regulating target gene expression by binding to the 3 -UTRs of their target mRNAs, and miRNAs are involved in negatively regulating gene expression at the posttranscriptional level (13). Bioinformatic analysis was performed using the program TargetScan, which found that NR4A2 contained a putative binding site for miR-183 in its 3'UTR. Moreover, it was demonstrated that miR-183 inhibitor transfection increased NR4A2 luciferase activity, as evaluated by a luciferase reporter assay, whereas the aforementioned effect was abolished when the predicted binding sites within the NR4A2 3'UTR were mutated. Importantly, NR4A2 Mrna and protein expression were also significantly increased in BMDMs transfected with the miR-183 inhibitor. A previous study demonstrated that members of the NR4A subfamily (NR4A1/Nur77, NR4A2/Nurr1 and NR4A3/Nor1) of orphan NRs (nuclear receptors) act as ligand-independent and constitutively active receptors, which are tightly controlled at the levels of expression, posttranslational modification and subcellular localization (41). NR4A subfamily members have emerged as key transcriptional regulators of cytokine and growth factor 
action in various diseases. Recently, accumulating evidence has suggested that NR4A nuclear receptors play pivotal roles in immunity and atherosclerosis (41), while all three NR4A family members are expressed within atherosclerotic lesions. The orphan nuclear receptor NR4A1 attenuates atherosclerosis by downregulating inflammatory gene expression in macrophages $(42,43)$. NR4A3 deficiency has been shown to inhibit vascular injury and atherosclerosis in mice $(44,45)$. NR4A2 has been shown to act as a repressor of NF-Kb activation in microglia and astrocytes and to attenuate atherosclerosis in mice (46). Exogenous expression of NR4A2 in macrophages leads to polarization toward their alternative phenotype, with induction of genes that are prototypical M2 markers (47). Moreover, NR4A2 transcriptionally activates Arg1 expression by directly binding to its promoter (7). Thus, it is speculated that miR-183 knockdown attenuated macrophage activation partially by upregulating NR4A2 expression, which may play an important protective role against atherogenesis.

In conclusion, the present study demonstrated that miR-183 attenuated macrophage activation contributed by decreasing the classic pro-inflammatory M1 macrophage but increasing alternative inflammation resolving M2 macrophage activation, accompanied by attenuation of foam-cell formation, along with enhanced genes associated with cholesterol efflux compared to uptake. This study highlights the important role of neutralizing miR-183 as a promising therapeutic strategy for atherosclerosis management.

\section{Acknowledgements}

Not applicable.

\section{Funding}

This work was supported by grants from the Science and Technology Fund of Health commission of Guizhou province (grant no. gzwjkj2019-1-038).

\section{Availability of data and materials}

The datasets used and/or analyzed during the current study are available from the corresponding author on reasonable request.

\section{Authors' contributions}

FHG and LL designed and conducted in vitro experiments, analyzed the data and drafted the paper. YSY and DHS performed the biomolecule experiment. YSZ, XSW and XPZ performed the pathological study and analyzed the data. XQX designed all of the experiments, supervised and funded the study, and contributed to the data analysis and to the writing of the paper. All authors read and approved the final manuscript and FHG and XQX confirm the authenticity of all the raw data.

\section{Ethics approval and consent to participate}

The animal study procedures were performed in accordance with the National Institutes of Health Guide for the Care and Use of Laboratory Animals and were approved by the Animal
Care and Use Committee of the Tongren municipal people's Hospital (approval no. 20190609).

\section{Patient consent for publication}

Not applicable.

\section{Competing interests}

The authors declare that they have no competing interests.

\section{References}

1. Libby P: Inflammation in atherosclerosis. Nature 420: 868-874, 2002.

2. Virani SS, Alonso A, Benjamin EJ, Bittencourt MS, Callaway CW, Carson AP, Chamberlain AM, Chang AR, Cheng S, Delling FN, et al: Heart disease and stroke statistics-2020 update: A report from the American heart association. Circulation 141: e139-e596, 2020.

3. Tabas I and Bornfeldt KE: Macrophage phenotype and function in different stages of atherosclerosis. Circ Res 118: 653-667, 2016.

4. Murray PJ: Macrophage polarization. Annu Rev Physiol 79: 541-566, 2017.

5. Mantovani A, Garlanda C and Locati M: Macrophage diversity and polarization in atherosclerosis: A question of balance. Arterioscler Thromb Vasc Biol 29: 1419-1423, 2009.

6. Sica A and Mantovani A: Macrophage plasticity and polarization: In vivo veritas. J Clin Invest 122: 787-795, 2012.

7. Leitinger $N$ and Schulman IG: Phenotypic polarization of macrophages in atherosclerosis. Arterioscler Thromb Vasc Biol 33: 1120-1126, 2013.

8. Tall AR, Yvan-Charvet L, Terasaka N, Pagler T and Wang N: HDL, ABC transporters, and cholesterol efflux: Implications for the treatment of atherosclerosis. Cell Metab 7: 365-375, 2008.

9. Tall AR and Yvan-Charvet L: Cholesterol, inflammation and innate immunity. Nat Rev Immunol 15: 104-116, 2015.

10. Gleissner CA, Shaked I, Little KM and Ley K: CXC chemokine ligand 4 induces a unique transcriptome in monocyte-derived macrophages. J Immunol 184: 4810-4818, 2010.

11. Finn AV, Nakano M, Polavarapu R, Karmali V, Saeed O, Zhao X, Yazdani S, Otsuka F, Davis T, Habib A, et al: Hemoglobin directs macrophage differentiation and prevents foam cell formation in human atherosclerotic plaques. J Am Coll Cardiol 59: 166-177, 2012.

12. Tabas I: Macrophage death and defective inflammation resolution in atherosclerosis. Nat Rev Immunol 10: 36-46, 2010.

13. Bartel DP: MicroRNAs: Genomics, biogenesis, mechanism, and function. Cell 116: 281-297, 2004.

14. Xu P, Guo M and Hay BA: MicroRNAs and the regulation of cell death. Trends Genet 20: 617-624, 2004.

15. Divakaran V and Mann DL: The emerging role of microRNAs in cardiac remodeling and heart failure. Circ Res 103: 1072-1083, 2008.

16. Bartel DP: MicroRNAs: Target recognition and regulatory functions. Cell 136: 215-233, 2009.

17. Yang CL, Zheng XL, Ye K, Ge H, Sun YN, Lu YF and Fan QX: MicroRNA-183 Acts as a tumor suppressor in human non-small cell lung cancer by down-regulating MTA1. Cell Physiol Biochem 46: 93-106, 2018.

18. Yang X, Wang L, Wang Q, Li L, Fu Y and Sun J: miR-183 inhibits osteosarcoma cell growth and invasion by regulating LRP6-Wnt/ק-catenin signaling pathway. Biochem Biophys Res Commun 496: 1197-1203, 2018.

19. Wei C, Song H, Sun X, Li D, Song J, Hua K and Fang L: miR-183 regulates biological behavior in papillary thyroid carcinoma by targeting the programmed cell death 4. Oncol Rep 34: 211-220, 2015.

20. Chen C, Ponnusamy M, Liu C, Gao J, Wang K and Li P: MicroRNA as a therapeutic target in cardiac remodeling. Biomed Res Int 2017: 1278436, 2017.

21. Zhang Y, Zhan Y, Liu D and Yu B: Inhibition of microRNA-183 expression resists human umbilical vascular endothelial cells injury by upregulating expression of IRS1. Drug Deliv 26: 612-621, 2019. 
22. Kim M, Jo H, Kwon Y, Kim Y, Jung HS and Jeoung D Homoharringtonine inhibits allergic inflammations by regulating NF-кB-miR-183-5p-BTG1 axis. Front Pharmacol 11: 1032, 2020.

23. Krausgruber T, Blazek K, Smallie T, Alzabin S, Lockstone H, Sahgal N, Hussell T, Feldmann M and Udalova IA: IRF5 promotes inflammatory macrophage polarization and TH1-TH17 responses. Nat Immunol 12: 231-238, 2011.

24. Liao X, Sharma N, Kapadia F, Zhou G, Lu Y, Hong H, Paruchuri K, Mahabeleshwar GH, Dalmas E, Venteclef N, et al: Kruppel-like factor 4 regulates macrophage polarization. J Clin Invest 121: 2736-2749, 2011.

25. Liu H, Cheng WL, Jiang X, Wang PX, Fang C, Zhu XY, Huang Z, She ZG and Li H: Ablation of interferon regulatory factor 3 protects against atherosclerosis in apolipoprotein E-deficient mice. Hypertension 69: 510-520, 2017.

26. Wang PX, Ji YX, Zhang XJ, Zhao LP, Yan ZZ, Zhang P, Shen LJ, Yang X, Fang J, Tian S, et al: Targeting CASP8 and FADD-like apoptosis regulator ameliorates nonalcoholic steatohepatitis in mice and nonhuman primates. Nat Med 23: 439-449, 2017.

27. Livak KJ and Schmittgen TD: Analysis of relative gene expression data using real-time quantitative PCR and the 2(-Delta Delta C(T)) method. Methods 25: 402-408, 2001.

28. Arner P and Kulyte A: MicroRNA regulatory networks in human adipose tissue and obesity. Nat Rev Endocrinol 11:276-288, 2015

29. Nazari-Jahantigh M, Egea V, Schober A and Weber C: MicroRNA-specific regulatory mechanisms in atherosclerosis. J Mol Cell Cardiol 89: 35-41, 2015.

30. SchoberA,Nazari-JahantighMandWeberC:MicroRNA-mediated mechanisms of the cellular stress response in atherosclerosis. Nat Rev Cardiol 12: 361-374, 2015.

31. Tana C, Giamberardino MA and Cipollone F: microRNA profiling in atherosclerosis, diabetes, and migraine. Ann Med 49: 93-105, 2017

32. Wang $\mathrm{H}$ and Cai $\mathrm{J}$ : The role of microRNAs in heart failure Biochim Biophys Acta Mol Basis Dis 1863: 2019-2030, 2017

33. Zhang L, Miller D, Yang Q and Wu B: MicroRNA regulatory networks as biomarkers in obesity: The emerging role. Methods Mol Biol 1617: 241-260, 2017.

34. Li C, Qu L, Farragher C, Vella A and Zhou B: MicroRNA regulated macrophage activation in obesity. J Transl Int Med 7: 46-52, 2019.

35. Lusis AJ: Atherosclerosis. Nature 407: 233-241, 2000.

36. Banerjee S, Xie N, Cui H, Tan Z, Yang S, Icyuz M, Abraham E and Liu G: MicroRNA let-7c regulates macrophage polarization. J Immunol 190: 6542-6549, 2013.
37. Ouimet M, Ediriweera HN, Gundra UM, Sheedy FJ, Ramkhelawon B, Hutchison SB, Rinehold K, van Solingen C, Fullerton MD, Cecchini K, et al: MicroRNA-33-dependent regulation of macrophage metabolism directs immune cell polarization in atherosclerosis. J Clin Invest 125: 4334-4348, 2015.

38. Ying W, Tseng A, Chang RC, Morin A, Brehm T, Triff K, Nair V, Zhuang G, Song H, Kanameni S, et al: MicroRNA-223 is a crucial mediator of PPAR $\gamma$-regulated alternative macrophage activation. J Clin Invest 125: 4149-4159, 2015

39. Qiu M, Ma J, Zhang J, Guo X, Liu Q and Yang Z: MicroRNA-150 deficiency accelerates intimal hyperplasia by acting as a novel regulator of macrophage polarization. Life Sci 240: 116985, 2020.

40. Takeuchi H, Tanaka M, Tanaka A, Tsunemi A and Yamamoto H: Predominance of M2-polarized macrophages in bladder cancer affects angiogenesis, tumor grade and invasiveness. Oncol Lett 11: 3403-3408, 2016.

41. McMorrow JP and Murphy EP: Inflammation: A role for NR4A orphan nuclear receptors? Biochem Soc Trans 39: 688-693, 2011.

42. Pei L, Castrillo A and Tontonoz P: Regulation of macrophage inflammatory gene expression by the orphan nuclear receptor Nur77. Mol Endocrinol 20: 786-794, 2006.

43. Hanna RN, Shaked I, Hubbeling HG, Punt JA, Wu R, Herrley E, Zaugg C, Pei H, Geissmann F, Ley K and Hedrick CC: NR4A1 (Nur77) deletion polarizes macrophages toward an inflammatory phenotype and increases atherosclerosis. Circ Res 110: 416-427, 2012.

44. Qing H, Liu Y, Zhao Y, Aono J, Jones KL, Heywood EB, Howatt D, Binkley CM, Daugherty A, Liang Y and Bruemmer D: Deficiency of the NR4A orphan nuclear receptor NOR1 in hematopoietic stem cells accelerates atherosclerosis. Stem Cells 32: 2419-2429, 2014

45. Qing H, Jones KL, Heywood EB, Lu H, Daugherty A and Bruemmer D: Deletion of the NR4A nuclear receptor NOR1 in hematopoietic stem cells reduces inflammation but not abdominal aortic aneurysm formation. BMC Cardiovasc Disord 17: 271, 2017.

46. Hamers AA, Hanna RN, Nowyhed H, Hedrick CC and de Vries CJ: NR4A nuclear receptors in immunity and atherosclerosis. Curr Opin Lipidol 24: 381-385, 2013.

47. Mahajan S, Saini A, Chandra V, Nanduri R, Kalra R, Bhagyaraj E, Khatri N and Gupta P: Nuclear Receptor Nr4a2 promotes alternative polarization of macrophages and confers protection in sepsis. J Biol Chem 290: 18304-18314, 2015. 